\title{
Editorial of Dynamical Systems, Control and Optimization
}

\author{
Changzhi Wu ${ }^{1} \cdot$ Kok Lay Teo ${ }^{1}$
}

Published online: 6 January 2018

(C) Foundation for Scientific Research and Technological Innovation 2018

Many practical significant real world problems are modelled as appropriate dynamical systems. Examples include micro-biological processes, chemical processes, aeronautical systems, and social economic systems. The control and optimization of these real world problems are challenging and practically importance. Due to the nonlinear nature of the problems, the formulated dynamical systems are usually highly nonlinear. To understand the behaviour of these problems, we first need to studied well posedness of proposed dynamical systems. Then, the corresponding control problems will be solved either through dynamical analysis or numerical computation.

This special issue is devoted to solving practical problems through new modelling techniques and state-of-the-art numerical optimization methods developed based on solid mathematical analysis. Several submissions were received and after thorough review process 15 research manuscripts were accepted for publication in this issue. Three of them are dedicated to study existence of solutions. Two of them are on numerical computation of solutions. The left is on study of dynamical systems, including system stability, controllability and solution behaviour.

We are greatly appreciated kind support from the editorial team during the preparation of this special issue, particularly Professor V. Sree Hari Rao, Editor-in-Chief for his valuable suggestions and comments. We also appreciate the support of Springer Publisher and the reviewers whose valuable comments and suggestions contribute to select the published papers in this issue.

\footnotetext{
Changzhi Wu

C.Wu@exchange.curtin.edu.au
}

1 Curtin University, Perth, Australia 